\title{
A REVERSE HILBERT-TYPE INEQUALITY WITH A GENERALIZED HOMOGENEOUS KERNEL
}

\author{
BING HE
}

Abstract. In this paper, by introducing a generalized homogeneous kernel and estimating the weight function, a new reverse Hilbert-type integral inequality with some parameters and a best constant factor is established. Furthermore, the corresponding equivalent form is considered.

\section{Introduction}

$$
\begin{aligned}
& \text { If } a_{n}, b_{n} \geq 0,0<\sum_{n=1}^{\infty} a_{n}^{2}<\infty \text { and } 0<\sum_{n=1}^{\infty} b_{n}^{2}<\infty \text {, then(see [1]) } \\
& \qquad \sum_{n=1}^{\infty} \sum_{m=1}^{\infty} \frac{a_{m} b_{n}}{m+n}<\pi\left(\sum_{n=1}^{\infty} a_{n}^{2}\right)^{1 / 2}\left(\sum_{n=1}^{\infty} b_{n}^{2}\right)^{1 / 2},
\end{aligned}
$$

where the constant factor $\pi$ is the best possible. Inequality (1.1) is well known as Hilbert's inequality. It is important in analysis and its applications. It was studied extensively and refinements, generalizations and numerous variants appeared in the literature (see [1]- [3]). Firstly, we shall recall some Hilbert's inequalities. If $f, g \geq 0, p>1, \frac{1}{p}+\frac{1}{q}=1,0<\int_{0}^{\infty} f^{p}(x) \mathrm{d} x<$ $\infty$ and $0<\int_{0}^{\infty} g^{q}(x) \mathrm{d} x<\infty$, then

$$
\int_{0}^{\infty} \int_{0}^{\infty} \frac{f(x) g(y)}{x+y} \mathrm{~d} x \mathrm{~d} y<\frac{\pi}{\sin (\pi / p)}\left\{\int_{0}^{\infty} f^{p}(x) \mathrm{d} x\right\}^{1 / p}\left\{\int_{0}^{\infty} g^{q}(x) \mathrm{d} x\right\}^{1 / q} ;
$$

where the constant factor $\frac{\pi}{\sin (\pi / p)}$ is the best possible. Inequality (1.2) is named of HardyHilbert's integral inequality (see [1]). Under the same condition of (1.2), we have the HardyHilbert's type inequality (see [1, Th. 319, Th. 341]) as:

$$
\int_{0}^{\infty} \int_{0}^{\infty} \frac{f(x) g(y)}{\max \{x, y\}} \mathrm{d} x \mathrm{~d} y<p q\left\{\int_{0}^{\infty} f^{p}(x) \mathrm{d} x\right\}^{1 / p}\left\{\int_{0}^{\infty} g^{q}(x) \mathrm{d} x\right\}^{1 / q}
$$

where the constant factor $p q$ is also the best possible. The corresponding inequality for series is :

$$
\sum_{n=1}^{\infty} \sum_{m=1}^{\infty} \frac{a_{m} b_{n}}{m+n}<\frac{\pi}{\sin (\pi / p)}\left(\sum_{n=1}^{\infty} a_{n}^{p}\right)^{1 / p}\left(\sum_{n=1}^{\infty} b_{n}^{q}\right)^{1 / q} ;
$$

Received September 18, 2009, revised March 302010.

2000 Mathematics Subject Classification. 26D15.

Key words and phrases. Hilbert's integral inequality, Weight function, reverse Hölder's inequality. 


$$
\sum_{n=1}^{\infty} \sum_{m=1}^{\infty} \frac{a_{m} b_{n}}{\max \{m, n\}}<p q\left(\sum_{n=1}^{\infty} a_{n}^{p}\right)^{1 / p}\left(\sum_{n=1}^{\infty} b_{n}^{q}\right)^{1 / q}
$$

where the constant factors $\frac{\pi}{\sin (\pi / p)}$ and $p q$ are both the best possible.

In 2007, Yang (see [4]) gave a reverse inequality as follows: If $0<p<1, \frac{1}{p}+\frac{1}{q}=1, \alpha>$ $0,2-p<\lambda<2-q, f(x), g(x) \geq 0$, such that $0<\int_{0}^{\infty} \frac{\left(x^{1-\alpha} f(x)\right)^{p}}{x^{1+\alpha(\lambda-2)}} \mathrm{d} x<\infty, 0<\int_{0}^{\infty} \frac{\left(x^{1-\alpha} g(x)\right)^{q}}{x^{1+\alpha(\lambda-2)}} \mathrm{d} x<\infty$ Then

$$
\int_{0}^{\infty} \int_{0}^{\infty} \frac{f(x) g(y)}{\left(x^{\alpha}+y^{\alpha}\right)^{\lambda}} \mathrm{d} x \mathrm{~d} y>\frac{1}{\alpha} k_{\lambda}(p)\left\{\int_{0}^{\infty} \frac{\left(x^{1-\alpha} f(x)\right)^{p}}{x^{1+\alpha(\lambda-2)}} \mathrm{d} x\right\}^{\frac{1}{p}}\left\{\int_{0}^{\infty} \frac{\left(x^{1-\alpha} g(x)\right)^{q}}{x^{1+\alpha(\lambda-2)}} \mathrm{d} x\right\}^{\frac{1}{q}},
$$

where the constant factor $\frac{1}{\alpha} k_{\lambda}(p)\left(k_{\lambda}(p)=B\left(\frac{p+\lambda-2}{\lambda p}, \frac{q+\lambda-2}{\lambda q}\right)\right)$ is the best possible. And in 2008, Xie (see [5]) got the following reverse form: For $0<p<1, a, b>0, a \neq b$ and some other conditions, then

$$
\int_{0}^{\infty} \int_{0}^{\infty} \frac{f(x) g(y)}{(x+a y)^{2}(x+b y)^{2}} \mathrm{~d} x \mathrm{~d} y>K\left\{\int_{0}^{\infty} \frac{1}{x^{p+1}} f^{p}(x) \mathrm{d} x\right\}^{1 / p}\left\{\int_{0}^{\infty} \frac{1}{x^{q+1}} g^{q}(x) \mathrm{d} x\right\}^{1 / q},
$$

where the constant factor $K:=\frac{a+b}{(b-a)^{2}}\left[\frac{\ln (b / a)}{b-a}-\frac{2}{a+b}\right]$ is the best possible.. By the way, in recent years, the reverse form of the Hardy-Hilbert's inequality has been studied by Zhao(see [6]) and other mathematicians.

Until now, we only focus on the Hilbert's inequality with $\lambda$ homogeneous and non-homogeneous kernel, but we are just at the beginning of the long trip on the study of the real number homogeneous kernel. Lots of related results will appear in the coming future.

The main purpose of this article is to establish the reverse form of the Hilbert's type inequality with the mixed homogeneous kernel of real number degree. We hope that this work will pave the way for the future research of the Hilbert's inequality.

\section{Main results}

Lemma 1. Setting $\lambda, \mu \in \mathbb{R}$ and $\lambda+\mu>0$, define the weight function $\varpi_{\lambda, \mu}(u)$ as

$$
\varpi_{\lambda, \mu}(u):=\int_{0}^{\infty} \frac{(\min \{u, v\})^{\lambda}}{(\max \{u, v\})^{\mu}} \cdot \frac{u^{-\frac{\lambda-\mu}{2}}}{v^{1+\frac{\lambda-\mu}{2}}} \mathrm{~d} v, u \in(0, \infty),
$$

then for $u \in(0, \infty)$, we have

$$
\varpi_{\lambda, \mu}(u)=\frac{4}{\lambda+\mu}, u \in(0, \infty) .
$$

Proof. For fixed $u>0$, setting $t=\frac{v}{u}$, we have

$$
\varpi_{\lambda, \mu}(u)=\int_{0}^{\infty} \frac{(\min \{u, v\})^{\lambda}}{(\max \{u, v\})^{\mu}} \cdot \frac{u^{-\frac{\lambda-\mu}{2}}}{v^{1+\frac{\lambda-\mu}{2}}} \mathrm{~d} v=\int_{0}^{\infty} \frac{(\min \{1, t\})^{\lambda}}{(\max \{1, t\})^{\mu}} \cdot t^{-1-\frac{\lambda-\mu}{2}} \mathrm{~d} t
$$




$$
\begin{aligned}
& =\int_{0}^{1} \frac{t^{\lambda}}{1} \cdot t^{-1-\frac{\lambda-\mu}{2}} \mathrm{~d} t+\int_{1}^{\infty} \frac{1}{t^{\mu}} \cdot t^{-1-\frac{\lambda-\mu}{2}} \mathrm{~d} t \\
& =\frac{4}{\lambda+\mu} .
\end{aligned}
$$

It follows that $\varpi_{\lambda, \mu}(u)=\frac{4}{\lambda+\mu}$, thus (2.2) is correct.

In what follows, $\lambda$, $\mu$ will be real numbers such that $\lambda+\mu>0$ and $p, q$ will be real numbers such that $-\infty<q<0,0<p<1$ and $\frac{1}{p}+\frac{1}{q}=1$.

Theorem 2. If $f, g \geq 0$ such that $0<\int_{0}^{\infty} x^{p\left(1+\frac{\lambda-\mu}{2}\right)-1} f^{p}(x) \mathrm{d} x<\infty$ and $0<\int_{0}^{\infty} x^{q\left(1+\frac{\lambda-\mu}{2}\right)-1} g^{q}(x)$ $\mathrm{d} x<\infty$, then we have the following inequality

$$
\begin{aligned}
I & :=\int_{0}^{\infty} \int_{0}^{\infty} \frac{(\min \{x, y\})^{\lambda}}{(\max \{x, y\})^{\mu}} f(x) g(y) \mathrm{d} x \mathrm{~d} y \\
& >\frac{4}{\lambda+\mu}\left\{\int_{0}^{\infty} x^{p\left(1+\frac{\lambda-\mu}{2}\right)-1} f^{p}(x) \mathrm{d} x\right\}^{1 / p}\left\{\int_{0}^{\infty} y^{q\left(1+\frac{\lambda-\mu}{2}\right)-1} g^{q}(y) \mathrm{d} y\right\}^{1 / q},
\end{aligned}
$$

where the constant factor $\frac{4}{\lambda+\mu}$ is independent of $p, q$ and $\frac{4}{\lambda+\mu}$ is the best possible.

Proof. By the reverse Hölder's inequality with weight[7], we obtain

$$
\begin{aligned}
& \int_{0}^{\infty} \int_{0}^{\infty} \frac{(\min \{x, y\})^{\lambda}}{(\max \{x, y\})^{\mu}} f(x) g(y) \mathrm{d} x \mathrm{~d} y \\
= & \int_{0}^{\infty} \int_{0}^{\infty} \frac{(\min \{x, y\})^{\lambda}}{(\max \{x, y\})^{\mu}}\left[\frac{x^{\left(1+\frac{\lambda-\mu}{2}\right) / q}}{y^{\left(1+\frac{\lambda-\mu}{2}\right) / p}} f(x)\right]\left[\frac{y^{\left(1+\frac{\lambda-\mu}{2}\right) / p}}{x^{\left(1+\frac{\lambda-\mu}{2}\right) / q}} g(y)\right] \mathrm{d} x \mathrm{~d} y \\
\geq & \left\{\int_{0}^{\infty} \int_{0}^{\infty} \frac{(\min \{x, y\})^{\lambda}}{(\max \{x, y\})^{\mu}} \frac{x^{(p-1)\left(1+\frac{\lambda-\mu}{2}\right)}}{y^{1+\frac{\lambda-\mu}{2}}} f^{p}(x) \mathrm{d} x \mathrm{~d} y\right\}^{1 / p} \\
& \times\left\{\int_{0}^{\infty} \int_{0}^{\infty} \frac{(\min \{x, y\})^{\lambda}}{(\max \{x, y\})^{\mu}} \frac{y^{(q-1)\left(1+\frac{\lambda-\mu}{2}\right)}}{x^{1+\frac{\lambda-\mu}{2}}} g^{q}(y) \mathrm{d} x \mathrm{~d} y\right\}^{1 / q} .
\end{aligned}
$$

If (2.4) takes the form of the equality, then there exist constants $a$ and $b$, such that they are not all zero and

$$
\frac{a x^{(p-1)\left(1+\frac{\lambda-\mu}{2}\right)}}{y^{1+\frac{\lambda-\mu}{2}}} \cdot f^{p}(x)=\frac{b y^{(q-1)\left(1+\frac{\lambda-\mu}{2}\right)}}{x^{1+\frac{\lambda-\mu}{2}}} \cdot g^{q}(y) \text { a.e. in }(0, \infty) \times(0, \infty) .
$$

Then we have $a x^{p\left(1+\frac{\lambda-\mu}{2}\right)} f^{p}(x)=b y^{q\left(1+\frac{\lambda-\mu}{2}\right)} g^{q}(y)$ a.e. in $(0, \infty) \times(0, \infty)$. Hence there exist a constant $c$, such that

$$
a x^{p\left(1+\frac{\lambda-\mu}{2}\right)} f^{p}(x)=b y^{q\left(1+\frac{\lambda-\mu}{2}\right)} g^{q}(y)=c \text { a.e. in }(0, \infty) \times(0, \infty) .
$$


Without losing the generality, suppose that $a \neq 0$, we may get $x^{p\left(1+\frac{\lambda-\mu}{2}\right)-1} f^{p}(x)=\frac{c}{a x}$ a.e. in $(0, \infty)$, which contradicts the fact that $0<\int_{0}^{\infty} x^{p\left(1+\frac{\lambda-\mu}{2}\right)-1} f^{p}(x) \mathrm{d} x<\infty$. Hence by (2.1), (2.3) takes a strict inequality as follows:

$$
\begin{aligned}
& \int_{0}^{\infty} \int_{0}^{\infty} \frac{(\min \{x, y\})^{\lambda}}{(\max \{x, y\})^{\mu}} f(x) g(y) \mathrm{d} x \mathrm{~d} y \\
> & \left\{\int_{0}^{\infty} \varrho_{\lambda, \mu}(x) x^{p\left(1+\frac{\lambda-\mu}{2}\right)-1} f^{p}(x) \mathrm{d} x\right\}^{\frac{1}{p}}\left\{\int_{0}^{\infty} \varpi_{\lambda, \mu}(y) y^{q\left(1+\frac{\lambda-\mu}{2}\right)-1} g^{q}(y) \mathrm{d} y\right\}^{\frac{1}{q}}
\end{aligned}
$$

In view of (2.2), we have (2.3).

Assume that the constant factor $\frac{4}{\lambda+\mu}$ in (2.3) is not the best possible, then there exists a positive number $k$ with $k>\frac{4}{\lambda+\mu}$ and $a>0$, such that

$$
\begin{aligned}
\int_{a}^{\infty} & {\left[\int_{0}^{\infty} \frac{(\min \{x, y\})^{\lambda}}{(\max \{x, y\})^{\mu}} f(x) g(y) \mathrm{d} y\right] \mathrm{d} x } \\
& >k\left(\int_{a}^{\infty} x^{p\left(1+\frac{\lambda-\mu}{2}\right)-1} f^{p}(x) \mathrm{d} x\right)^{\frac{1}{p}}\left(\int_{a}^{\infty} y^{q\left(1+\frac{\lambda-\mu}{2}\right)-1} g^{q}(y) \mathrm{d} y\right)^{\frac{1}{q}} .
\end{aligned}
$$

For $0<\varepsilon<\frac{(\lambda+\mu)|q|}{2}$, setting

$$
\widetilde{f}(x)=\left\{\begin{array}{ll}
0, & x \in(0, a), \\
x^{-\left(1+\frac{\lambda-\mu}{2}\right)-\frac{\varepsilon}{p}}, & x \in[a, \infty),
\end{array} \widetilde{g}(y)= \begin{cases}0, & y \in(0, a), \\
y^{-\left(1+\frac{\lambda-\mu}{2}\right)-\frac{\varepsilon}{q}}, & y \in[a, \infty) .\end{cases}\right.
$$

Putting $\widetilde{f}(x), \widetilde{g}(y)$ into (2.5). For fixed $y$, setting $t=\frac{y}{x}$, we find

$$
\begin{aligned}
\int_{a}^{\infty} & {\left[\int_{0}^{\infty} \frac{(\min \{x, y\})^{\lambda}}{(\max \{x, y\})^{\mu}} \widetilde{f}(x) \widetilde{g}(y) \mathrm{d} y\right] \mathrm{d} x } \\
& \leq \int_{a}^{\infty} x^{-1-\varepsilon}\left[\int_{0}^{\infty} \frac{(\min \{1, t\})^{\lambda}}{(\max \{1, t\})^{\mu}} \cdot t^{-1-\frac{\lambda-\mu}{2}-\frac{\varepsilon}{q}} \mathrm{~d} t\right] \mathrm{d} x \\
& =\frac{1}{\varepsilon a^{\varepsilon}}\left(\frac{1}{\frac{\lambda+\mu}{2}-\frac{\varepsilon}{q}}+\frac{1}{\frac{\lambda+\mu}{2}+\frac{\varepsilon}{q}}\right) .
\end{aligned}
$$

Multiplying by $\varepsilon a^{\varepsilon}$ for both sides of (2.5), by the above inequality, we obtain

$$
\begin{aligned}
& \frac{1}{\frac{\lambda+\mu}{2}-\frac{\varepsilon}{q}}+\frac{1}{\frac{\lambda+\mu}{2}+\frac{\varepsilon}{q}} \geq \varepsilon a^{\varepsilon} \int_{a}^{\infty}\left[\int_{0}^{\infty} \frac{(\min \{x, y\})^{\lambda}}{(\max \{x, y\})^{\mu}} \widetilde{f}(x) \widetilde{g}(y) \mathrm{d} y\right] \mathrm{d} x \\
& >\varepsilon a^{\varepsilon} k\left(\int_{a}^{\infty} x^{p\left(1+\frac{\lambda-\mu}{2}\right)-1} \widetilde{f}^{p}(x) \mathrm{d} x\right)^{\frac{1}{p}}\left(\int_{a}^{\infty} y^{q\left(1+\frac{\lambda-\mu}{2}\right)-1} \widetilde{g}^{q}(y) \mathrm{d} y\right)^{\frac{1}{q}}=k .
\end{aligned}
$$

It follows that $\frac{4}{\lambda+\mu} \geq k\left(\varepsilon \rightarrow 0^{+}\right)$, which contradicts the hypothesis. Hence the constant factor $\frac{4}{\lambda+\mu}$ in (2.3) is the best possible. This completes the proof. 
Theorem 3. If $f \geq 0$ such that $0<\int_{0}^{\infty} x^{p\left(1+\frac{\lambda-\mu}{2}\right)-1} f^{p}(x) \mathrm{d} x<\infty$, then we have the following equivalent inequality of (2.3)

$$
\begin{aligned}
J & :=\int_{0}^{\infty} y^{-\frac{p(\lambda-\mu)}{2}-1}\left[\int_{0}^{\infty} \frac{(\min \{x, y\})^{\lambda}}{(\max \{x, y\})^{\mu}} f(x) \mathrm{d} x\right]^{p} \mathrm{~d} y \\
& >\left(\frac{4}{\lambda+\mu}\right)^{p} \int_{0}^{\infty} x^{p\left(1+\frac{\lambda-\mu}{2}\right)-1} f^{p}(x) \mathrm{d} x,
\end{aligned}
$$

where the constant factor $\left(\frac{4}{\lambda+\mu}\right)^{p}$ is the best possible.

Proof. Since $\int_{0}^{\infty} x^{p\left(1+\frac{\lambda-\mu}{2}\right)-1} f^{p}(x) \mathrm{d} x>0$, it is obvious that $J>0$. If $J=\infty$, then (2.6) is valid naturally. We now assume $J<\infty$ and $g(y)=y^{-1-\frac{p(\lambda-\mu)}{2}}\left[\int_{0}^{\infty} \frac{(\min \{x, y\})^{\lambda}}{(\max \{x, y\})^{\mu}} f(x) \mathrm{d} x\right]^{p-1}, y \in(0, \infty)$. By (2.3), we get

$$
\begin{aligned}
\infty & >\int_{0}^{\infty} y^{q\left(1+\frac{\lambda-\mu}{2}\right)-1} g^{q}(y) \mathrm{d} y=J=I \\
& >\frac{4}{\lambda+\mu}\left\{\int_{0}^{\infty} x^{p\left(1+\frac{\lambda-\mu}{2}\right)-1} f^{p}(x) \mathrm{d} x\right\}^{1 / p}\left\{\int_{0}^{\infty} y^{q\left(1+\frac{\lambda-\mu}{2}\right)-1} g^{q}(y) \mathrm{d} y\right\}^{1 / q}>0, \\
J^{1 / p} & =\left\{\int_{0}^{\infty} y^{q\left(1+\frac{\lambda-\mu}{2}\right)-1} g^{q}(y) \mathrm{d} y\right\}^{1 / p}>\frac{4}{\lambda+\mu}\left\{\int_{0}^{\infty} x^{p\left(1+\frac{\lambda-\mu}{2}\right)-1} f^{p}(x) \mathrm{d} x\right\}^{1 / p} .
\end{aligned}
$$

Hence (2.6) is valid.

On the other hand, suppose that (2.6) is valid. By the reverse Hölder's inequality with weight, we find

$$
\begin{aligned}
I & =\int_{0}^{\infty}\left[y^{-\frac{\lambda-\mu}{2}-\frac{1}{p}} \int_{0}^{\infty} f(x) \mathrm{d} x\right]\left[y^{\frac{\lambda-\mu}{2}+\frac{1}{p}} g(y)\right] \mathrm{d} y \\
& \geq J^{1 / p}\left\{\int_{0}^{\infty} y^{q\left(1+\frac{\lambda-\mu}{2}\right)-1} g^{q}(y) \mathrm{d} y\right\}^{1 / q} .
\end{aligned}
$$

Then by (2.6), we have (2.3). Thus (2.3) and (2.6) are equivalent.

It is of course that the constant factor in (2.6) is the best possible. Otherwise, by (2.7), we may get a contradiction that the constant factor in (2.3) is not the best possible. This completes the proof.

Theorem 4. If $g \geq 0$ such that $0<\int_{0}^{\infty} x^{q\left(1+\frac{\lambda-\mu}{2}\right)-1} g^{q}(x) \mathrm{d} x<\infty$, then we have the following equivalent inequality of (2.3)

$$
\begin{aligned}
L & :=\int_{0}^{\infty} x^{-\frac{q(\lambda-\mu)}{2}-1}\left[\int_{0}^{\infty} \frac{(\min \{x, y\})^{\lambda}}{(\max \{x, y\})^{\mu}} g(y) \mathrm{d} y\right]^{q} \mathrm{~d} x \\
& <\left(\frac{4}{\lambda+\mu}\right)^{q} \int_{0}^{\infty} y^{q\left(1+\frac{\lambda-\mu}{2}\right)-1} g^{q}(y) \mathrm{d} y,
\end{aligned}
$$

where the constant factor $\left(\frac{4}{\lambda+\mu}\right)^{q}$ is the best possible. 
Proof. If $L=0$, then (2.8) is valid naturally; If $L>0$, for $y \in(0, \infty)$, setting

$$
[g(y)]_{n}= \begin{cases}\frac{1}{n}, & g(y)<\frac{1}{n}, \\ g(y), & \frac{1}{n} \leq g(y) \leq n, \\ n, & g(y)>n,\end{cases}
$$

then there exists $n_{0} \in \mathbb{N}$ such that for all $n \geq n_{0}$, we have

$$
\begin{gathered}
\int_{\frac{1}{n}}^{n} y^{q\left(1+\frac{\lambda-\mu}{2}\right)-1}[g(y)]_{n}^{q} \mathrm{~d} y>0, \\
L(n):=\int_{\frac{1}{n}}^{n} x^{-\frac{q(\lambda-\mu)}{2}-1}\left(\int_{\frac{1}{n}}^{n} \frac{(\min \{x, y\})^{\lambda}}{(\max \{x, y\})^{\mu}}[g(y)]_{n} \mathrm{~d} y\right)^{q} \mathrm{~d} x>0 .
\end{gathered}
$$

Furthermore, supposing

$$
\begin{aligned}
f_{n}(x) & :=x^{-\frac{q(\lambda-\mu)}{2}-1}\left(\int_{\frac{1}{n}}^{n} \frac{(\min \{x, y\})^{\lambda}}{(\max \{x, y\})^{\mu}}[g(y)]_{n} \mathrm{~d} y\right)^{q-1}, \\
I(n) & :=\int_{\frac{1}{n}}^{n} \int_{\frac{1}{n}}^{n} \frac{(\min \{x, y\})^{\lambda}}{(\max \{x, y\})^{\mu}} f_{n}(x)[g(y)]_{n} \mathrm{~d} x \mathrm{~d} y\left(n \geq n_{0}\right) .
\end{aligned}
$$

By (2.3), in view of $q<0$, we have

$$
\begin{aligned}
\infty & >\int_{\frac{1}{n}}^{n} x^{p\left(1+\frac{\lambda-\mu}{2}\right)-1} f_{n}^{p}(x) \mathrm{d} x=L(n)=I(n) \\
& >\frac{4}{\lambda+\mu}\left\{\int_{\frac{1}{n}}^{n} x^{p\left(1+\frac{\lambda-\mu}{2}\right)-1} f_{n}^{p}(x) \mathrm{d} x\right\}^{1 / p}\left\{\int_{\frac{1}{n}}^{n} y^{q\left(1+\frac{\lambda-\mu}{2}\right)-1}[g(y)]_{n}^{q} \mathrm{~d} y\right\}^{1 / q}>0, \\
0 & <\int_{\frac{1}{n}}^{n} x^{p\left(1+\frac{\lambda-\mu}{2}\right)-1} f_{n}^{p}(x) \mathrm{d} x=L(n)<\left(\frac{4}{\lambda+\mu}\right)^{q} \int_{0}^{\infty} y^{q\left(1+\frac{\lambda-\mu}{2}\right)-1} g^{q}(y) \mathrm{d} y<\infty .
\end{aligned}
$$

This shows that $0<\int_{0}^{\infty} x^{p\left(1+\frac{\lambda-\mu}{2}\right)-1} f_{\infty}^{p}(x) \mathrm{d} x<\infty$, when $n \rightarrow \infty$, applying (2.3), the above two inequalities still take the form of strict inequality. Hence (2.8) is correct.

On the other hand, assume that (2.8) is valid. By the reverse Hölder's inequality with weight, we find

$$
\begin{aligned}
I & =\int_{0}^{\infty}\left[x^{\frac{\lambda-\mu}{2}+\frac{1}{q}} f(x)\right]\left[x^{-\frac{\lambda-\mu}{2}-\frac{1}{q}} \int_{0}^{\infty} \frac{(\min \{x, y\})^{\lambda}}{(\max \{x, y\})^{\mu}} g(y) \mathrm{d} y\right] \mathrm{d} x \\
& \geq L^{1 / q}\left\{\int_{0}^{\infty} x^{p\left(1+\frac{\lambda-\mu}{2}\right)-1} f^{p}(x) \mathrm{d} x\right\}^{1 / p} .
\end{aligned}
$$

Then by (2.8), we have (2.3). Thus (2.8) and (2.3) are equivalent.

It is obviously that the constant factor in (2.8) is the best possible. Otherwise, by (2.9), we may get a contradiction that the constant factor in (2.3) is not the best possible. This completes the proof. 


\section{Acknowledgements}

The work was partially supported by the Emphases Natural Science Foundation of Guangdong Institution of Higher Learning, College and University (No. 05Z026). The authors would like to thank the anonymous referee for his or her suggestions and corrections.

\section{References}

[1] G. H. Hardy, J. E. Littlewood and G. Polya, Inequalities, Cambridge University Press, 1934.

[2] D. S. Mitrinovic, J. E. Pecaric and A. M. Fink, Inequalities Involving Functions and Their Integrals and Derivatives, Kluwer Academic, Boston, 1991.

[3] B. Yang, The Norm of Operator and Hilbert-type Inequalities. Science Press, Beijing, 2009.

[4] B. Yang, On a reverse Hardy-Hilbert's inequality, KYUNGPOOK Math. J., 47(2007), 411-423.

[5] Z. Xie and B. Yang, A new Hilbert-type integral inequality with some parameters and its reverse, Kyungpook Math. J., 48(2008), 93-100.

[6] C. Zhao and L. Debnath, Some new inverse type Hilbert integral inequalities, Journal of Mathematical Analysis and Applications, 262(2001), 411-418.

[7] J. Kuang, Applied Inequalities, Shangdong Science Press, Jinan, 2004.

Department of Mathematics, Guangdong Education College, Guangzhou, 510303 P. R. China.

E-mail: hzs314@163.com 